\title{
FIRST BEAM TESTS OF THE MUON COLLIDER TARGET TEST BEAM LINE AT THE AGS *
}

\author{
K.A. Brown, D. Gassner, J.W. Glenn, R. Prigl, N. Simos, J. Scaduto, N. Tsoupas, \\ Brookhaven National Laboratory, Upton, New York 11973, USA
}

\begin{abstract}
In this report we will describe the muon collider target test beam line which operates off one branch of the AGS switchyard. The muon collider target test facility is designed to allow a prototype muon collider target system to be developed and studied. The beam requirements for the facility are ambitious but feasible. The system is designed to accept bunched beams of intensities up to $1.6 \times 10^{13} 24$ $\mathrm{GeV}$ protons in a single bunch. The target specifications require beam spot sizes on the order of $1 \mathrm{~mm}, 1$ sigma rms at the maximum intensity. We will describe the optics design, the instrumentation, and the shielding design. Results from the commissioning of the beam line will be shown.
\end{abstract}

\section{INTRODUCTION}

The AGS switchyard has been in use for fixed target experiments for over two decades. During this time the beam intensity has been increased from $1 \times 10^{13}$ to over $7 \times 10^{13}$ protons per cycle. The fixed target area was designed, though, to accept slow extracted beam, which has significantly different characteristics from a fast beam pulse. In [1] was described the operation of the slow extracted beam for high intensity protons and the modifications required to accept the high intensity beams. In that report it was noted that the beam characteristics of the pre-Booster low intensity AGS and the post-Booster high intensity AGS were changed, changing the beam characteristics of the slow extracted beam. Most significantly the emittance of the beam was much larger in the post-Booster AGS. As a result, the matching section of the switchyard needed to be changed to match to a beam of larger area and larger momentum spread [1].

In recent years there has been an increasing demand to perform fast beam experiments. Two of these groups are interested in performing engineering studies for new target systems for the muon collider project [3] and for the neutron spallation project [4]. The beam requirements of these experiments are ambitious. Upon learning we could send a fast beam into the switchyard, [2] we needed to evaluate whether the switchyard could accept very high intensity fast beam pulses as high as $1.6 \times 10^{13}$ protons per (roughly $100 \mathrm{nsec}$ ) bunch. This has implications on the amount of existing shielding, instrumentation, on beam pipe windows, and on the magnetic optics of the beam transport.

${ }^{*}$ Work performed under the auspices of the U.S. Dept. of Energy.
It also had significant implications on the matching section. Fortunately, the modifications required to accept high intensity protons gave us the degrees of freedom to define new optics for the matching section, without having to move or replace any magnets. In addition existing shielding was more than adequate, requiring no changes.

In this report we will describe the optics for matching to the fast extracted beam. We will describe the instrumentation changes made to the fixed target areas. And we will present results of the commissioning and operation of the fast extracted beam to the muon collider target test area.

\section{DESCRIPTION OF BEAM TRANSPORT SYSTEM}

The AGS switchyard was designed to split slow extracted beams into as many as four separate beams, directed to individual beam lines through the use of electrostatic beam splitters and vertical Lambertson magnets. To effectively split the beams with minimum beam loss, the optics was designed to create a parallel beam through the splitter and Lambertson section. There are four quadrupoles for matching to the AGS at the very upstream section of the switchyard and a single quadrupole at the entrance to the A line, to bring vertical beam size down to fit into the large bending magnets. After the beam exits the 7.4 degree bend there are a set of quadrupoles whose purpose is to focus beam onto a target station (denoted ATGT near the middle of figure 1). With no target in place beam can continue down to the muon collider target location. Figure 1 shows the predicted $1 / 2$ beam sizes (horizontal on top, vertical on bottom) assuming a 120 pi-mm-mrad (normalized) particle, with a dp/p $=1 x 10^{-3}$. The final focus is a $1 \mathrm{~mm}^{2}$ at 1 sigma spot on the target.

\section{INITIAL EMITTANCE AND TWISS PARAMETERS}

The actual initial twiss parameters assumed in figure 1 depend on the trajectory of the beam as it exits the AGS. Using different models of the AGS we studied the trajectory of the beam as it passed through the edge fields of the AGS combined function magnets and developed a set of twiss parameters for our initial calculations. The differences between models were significant. Table 1 lists the modeled and measured twiss parameters. Not shown in figure 1 is the initial dispersion. The large error bars 


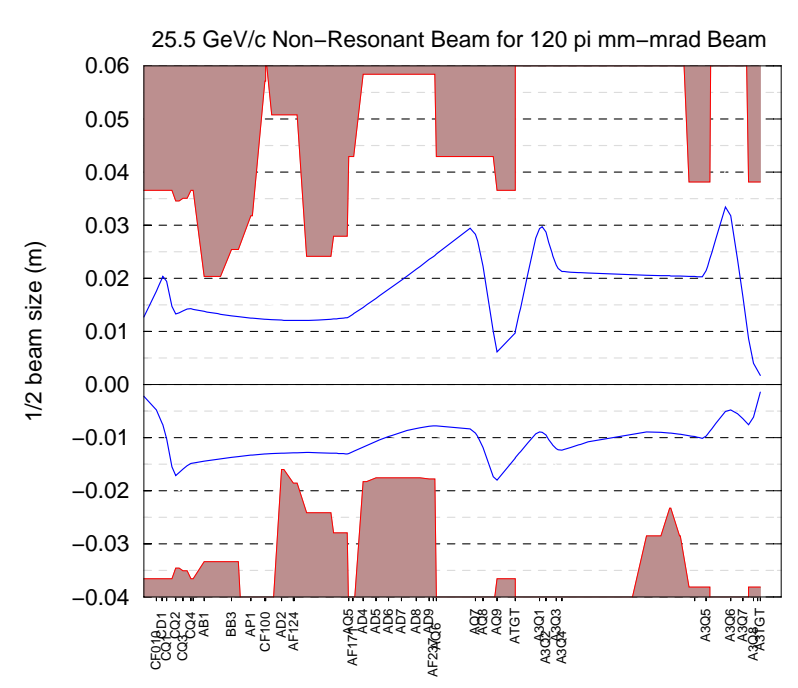

Figure 1: 1/2 horizontal and vertical beam sizes for transport to the muon collider target.

on the measured data are due to the difficulty in subtracting out momentum dispersion from the measured beam widths. The technique used to make these measurements was to vary the matching section quadrupoles and measure the beam widths on a flag (which we will denote CF100, an illumination screen utilizing a Dage 70R video camera and processing images with an Imaging Technology video frame grabber. See figure 5 for an example from the muon collider target flag). The main difficulty with this technique is the flag is not located in a dispersion free area, and momentum dispersion can be a significant factor in the beam size, for many of the quadrupole settings. For this data we had to select data points where the momentum dispersion terms could be subtracted, but were not a significant fraction of the beam size.

Table 1: Initial twiss parameters

\begin{tabular}{|l|c|c|c|}
\hline & MAD & field map tracking & measured \\
\hline$\beta_{H}(\mathbf{m})$ & 53 & 128 & $80 \pm 30$ \\
$\alpha_{H}$ & -6.7 & -17.9 & $-10 \pm 5$ \\
$\beta_{V}(\mathbf{m})$ & 2.2 & 4.1 & $3.97 \pm 1$ \\
$\alpha_{V}$ & 0.34 & 0.55 & $1.22 \pm 0.3$ \\
$\epsilon_{H}^{95 \%, N}$ & $100 \pi$ & $100 \pi$ & $90 \pm 50 \pi$ \\
$\epsilon_{V}^{95 \%, N}$ & $100 \pi$ & $100 \pi$ & $40 \pm 10 \pi$ \\
\hline
\end{tabular}

\section{MOMENTUM DISPERSION CONSIDERATIONS}

The momentum dispersion in a slow extracted beam is typically not a significant factor in the beam size, since it is spread out over the length of a beam spill, and so can be compensated for by ramping fields in magnets. The instantaneous momentum term in the slow spill is extremely small and can be ignored. For a fast extracted beam this is no longer true. The entire beam is only $100 \mathrm{nsec}$ long, and has significant momentum spread (as much as $3 x 10^{-3}$ for the large emittance high intensity beam). Beam size can be dominated by the momentum spread in the beam in many locations. In addition, although not strictly momentum dispersion, the beam size can also be effected by the fast kicker (whose pulse is a half sine wave, $300 \mathrm{nsec}$ at the base). We were careful to design the optics to give a zero momentum dispersion at the muon collider target location, but this was assuming a certain initial momentum dispersion for the beam line. In addition we were careful to design the optics to set the $R_{11}$ lump matrix term from the kicker to the target location to be as near zero as possible. We learned during beam tests that our assumed initial momentum dispersion was wrong. Figure 2 shows the predicted and measured dispersion at the CF100 flag location.

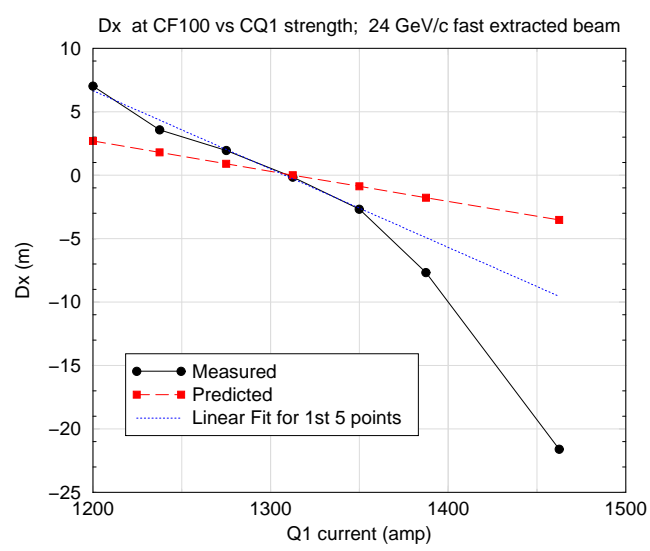

Figure 2: Measured and Predicted Momentum Dispersion at CF100.

\section{BEAM-LINE WINDOW ANALYSIS}

The response of a window structure to a focused, energetic beam and the potential failure due to the induced thermal shock is a problem we have investigated. For a detailed description of this analysis see [5] Assuming that thermalization of the material due to the energy left behind by the beam takes place instantly, then the initiators of the shock as well as its amplitude become the duration and the structure of the pulse. As the material thermalizes in the heated zone, an initial quasi-static stress develops. The material further responds by generating shock waves due to the stress gradients that exist between the heated and nonheated parts as well as between the front and back window surfaces. In the thin window structures, the propagation and reflection of the von Mises stresses between the two surfaces are the most important components that will determine whether the material will fail. In analyzing the A3 line and taking into account the time structure of the proton pulse, the protons in the pulse and the beam spot size at different locations along the line, an number of window 
candidate materials were examined. By pushing the beam to extremes, such as $1.6 \times 10^{13}$ protons per pulse with 0.5 $\mathrm{mm}$ rms sigma spot, very few, if any, materials can survive a single pulse. Shown in Figure 3 below is the resulting shock wave from such beam in a 10-mil thick Stainless Steel window. The resulting von Mises stresses of 2500 MPa are far above the yield and ultimate strengths of the material (800 and $900 \mathrm{MPa}$ respectively). Seen in the Figure are the reflections of the shock wave from the window edge. Other calculations show that aluminum windows will experience shock stresses beyond their strength limits for similarly intense beams with even a $1 \mathrm{~mm}$ rms sigma.

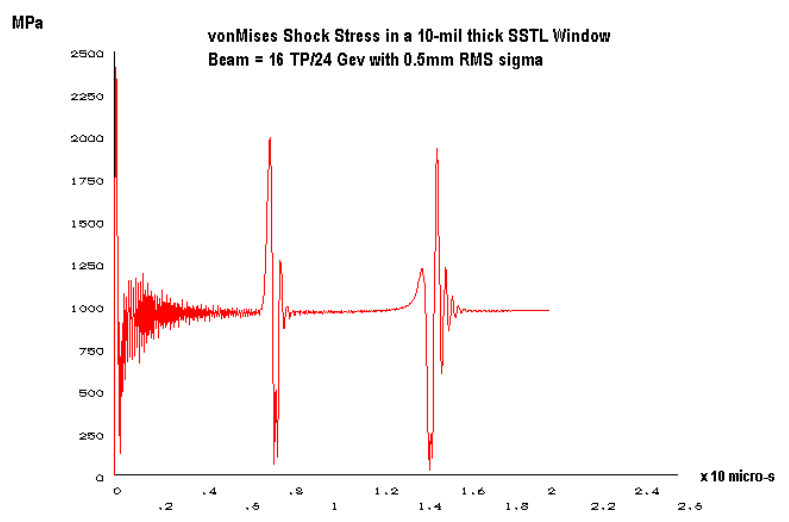

Figure 3: Shock stress in a 10-mil thick Stainless Steel window

\section{FY01 COMMISSIONING RESULTS}

During initial commissioning we found we could cleanly transport the lower intensity beam pulses, but as we raised intensity our efficiency dropped. As shown in [2] there is evidence of emittance growth. This is seen also in the beam transport. Figure 4 shows the final beam intensity measured in the experiment, as a function of extracted beam intensity. The design goal for beam size on target was $1 \mathrm{~mm}^{2}$ spot, which was achieved. Our models suggested we should be able to get a smaller spot, as small as $0.5 \mathrm{~mm}^{2}$, but we were not able to achieve this. The main reason for this was the momentum dispersion at the target was not zero, and for such small spots even a small amount of dispersion translates into a significant fraction of the beam size.

\section{CONCLUSIONS}

The muon collider target tests beam line has been very successfully commissioned and the experiment has been able to produce useful results from this years beam tests. We still have lots of work to do to get the design single bunch beam intensities. Future studies will focus on ways of getting high intensity single bunches down to the target, with minimal emittance growth.

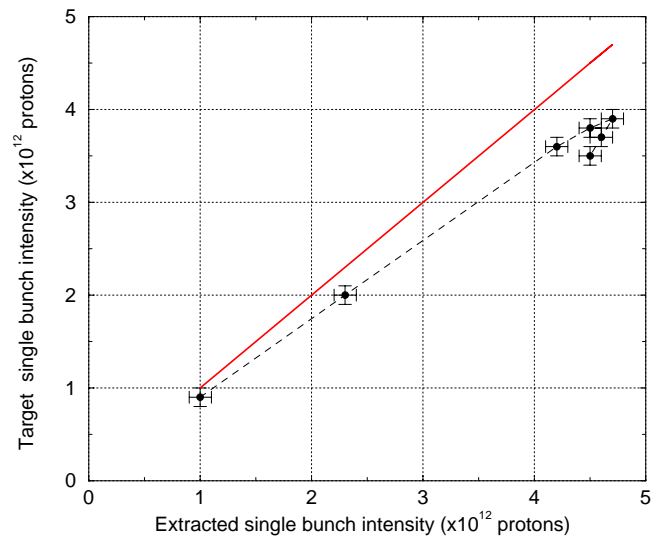

Figure 4: Target beam intensity vs extracted beam intensity. The red line corresponds to a $100 \%$ transmission, to guide the eye.

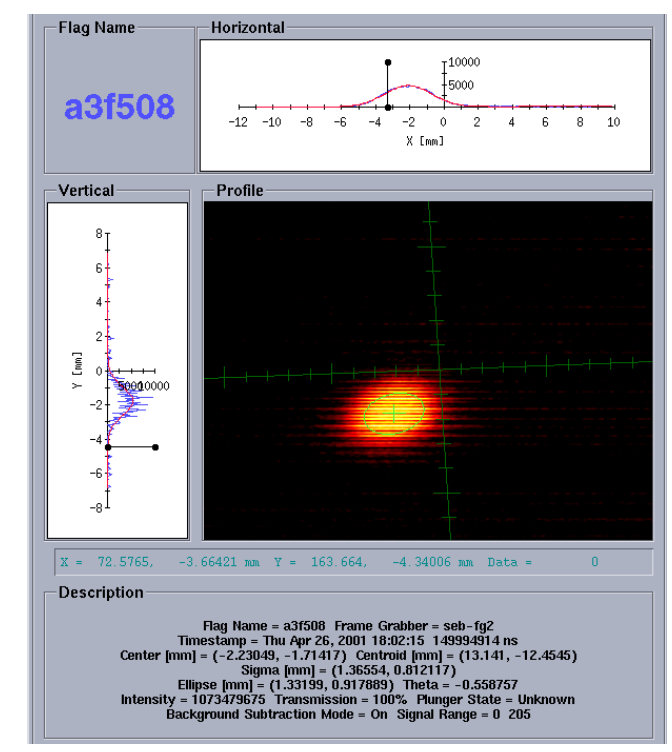

Figure 5: Beam spot on target.

\section{REFERENCES}

[1] K.A. Brown et al., "AGS Resonant Extraction with High Intensity Beams", Proceedings of the 1999 Particle Accelerator Conference, New York, New York (IEEE, Piscataway, NJ, 2000), pp. 3291-3293

[2] K.A. Brown et al., "Multiple Single Bunch Extraction to the AGS Switchyard", These proceedings.

[3] H. Kirk, et al, "Target Studies with BNL E951 at the AGS.", These Proceedings.

[4] T.A. Gabriel, et al, "Target Systems Overview for the Spallation Neutron Source”, Nuclear Technology, pp.49-60, Vol.32, October 2000.

[5] N.Simos et al, "Thermal Shock Analysis of Windows Interacting with Energetic, Focused Beam of the BNL Muon Target Experiment", These Proceedings. 